\title{
Condiciones laborales de los cirujanos
}

\section{Working conditions for surgeons}

El directorio de la SCCH, ha definido como una de las tareas prioritarias, el estudio de las condiciones en que ejercen su trabajo los cirujanos en servicios públicos y privados. Con la obtención de esta información, será posible avanzar en la definición de estándares mínimos para el ejercicio de la especialidad en un servicio de cirugía.

Para iniciar este trabajo, hemos desarrollado una encuesta que se hará llegar a los diferentes servicios. Para que los resultados sean significativos, es fundamental que sea respondida por un número importante de cirujanos. Los resultados se constituirán en el punto de partida para un diagnóstico; sobre la base de los resultados y con el respaldo de información significativa, se podrá definir las condiciones necesarias para el ejercicio de la especialidad en los servicios quirúrgicos.

Necesitamos un número importante de encuestas respondidas que respalden los resultados. El establecimiento de condiciones mínimas para el correcto ejercicio de la cirugía, con el aval de una sociedad científica de prestigio, como la nuestra, nos permitirá llevar con fuerza los planteamientos a las autoridades con el aval que otorga el respaldo de una información real.

Dr. Eduardo Coddou C.

Vicepresidente Sociedad de Cirujanos de Chile

Abril de 2013 